rev.relac.int.estrateg.segur.6(1):149-165,2011

\title{
GEOPOLÍTICA DE LOS RECURSOS ESTRATÉGICOS: CONFLICTOS POR AGUA EN AMÉRICA LATINA*
}

\author{
María Fernanda Ramírez ${ }^{* *}$ \\ María José Yepes ${ }^{* *}$
}

\section{RESUMEN}

Algunos recursos naturales como el agua son indispensables para la supervivencia humana, razón por la cual existe interés en apoderarse de ellos, o de obtener beneficio de su escasez. América Latina cuenta con grandes reservas de recursos hídricos, por lo que, se han presentado diversas situaciones conflictivas y malestar a nivel social, pero a su vez, se han delimitado los intereses de diversos actores del conflicto, como son los organismos internacionales de diferente orden, las transnacionales y los países desarrollados que han agotado o contaminado parte de sus reservas de agua.

Palabras clave: recursos estratégicos, conflicto, privatización, intervencionismo, agua.

* El presente ensayo es una reflexión académica presentada al término de la Cátedra de Política Económica Internacional, desarrollada en la Facultad de Economía de la Universidad Santo Tomás.

** Estudiante de último semestre de economía y tercer semestre de estadística de la Universidad Santo Tomás. Auxiliar de investigación, participante en el Semillero de Investigación del grupo Geopolítica y Relaciones Internacionales de la Facultad de Economía de la Universidad Santo Tomás.mafers23@hotmail.com

*** Estudiante de último semestre de economía de la Universidad Santo Tomás.mariajoseyepes@hotmail.com 


\begin{abstract}
Some natural resources, like water, are essential for human survival and for this reason interest exists in seizing them, or to obtain benefit from its shortage. Latin America has large reserves of water resources, for which, diverse troubling situations and unrest at the social level have existed; but, at the same time, limits has been placed on the interests of the various actors in the conflict, such as international organizations of various kinds, transnational corporations and developed countries, that have depleted or polluted part of its water reserves.
\end{abstract}

Key words: strategic resources, conflict, privatization, interventionism, water.

\title{
RESUMO
}

Alguns recursos naturais como a água são indispensáveis para a sobrevivência humana, razão pela qual existe interesse em apoderar-se deles ou de se obter benéficos por sua escassez. A América latina possui grandes reservas de recursos hídricos e, em consequência, vêm ocorrendo diversas situações conflitantes e de mal estar a nível social e, além disso, têm se delimitado os interesses de diversos atores do conflito, como os organismos internacionais de diferentes ordens, as transnacionais e os países desenvolvidos que têm esgotado e contaminado parte das suas reservas de água.

Palavras chave: recursos estratégicos, conflito, privatização, intervencionismo, água.

\section{INTRODUCCIÓN}

En la actualidad el agua se ha convertido en algo más que una fuente de vida; hoy en día el agua significa una fuente de conflictos, una guerra de poderes, una lucha por la supervivencia y una fuente de riqueza; la falta de acceso al vital líquido es un motivo de desigualdad, pobreza e injusticia social, como también una brecha más que diferencia a los países del tercer mundo respecto a los países desarrollados. La ineficiencia en el uso de este recurso por estos últimos se ha convertido en un límite a su crecimiento y a su bienestar, este límite es una consecuencia que no están dispuestos a asumir.

De acuerdo con el Segundo Informe sobre el Desarrollo de los Recursos Hídricos en el Mundo (2006), donde el estado del recurso, establecido por la UNESCO, se evidencia como un tema crítico porque el 2,5\% del agua potable que existe a nivel mundial se usa de manera ineficiente y se subdivide de la siguiente forma: el 70\% es utilizado para riego agrícola, el 10\% sirve a la industria y con el resto se produce electricidad (20\%). Además, tres millones de personas al año 
mueren de sed, una de cada tres en el mundo no tiene acceso al agua potable, lo que genera un aumento en la tasa de mortalidad por el consumo de aguas contaminadas.

Los organismos internacionales se han interesado por el estudio de las fuentes de recursos estratégicos como el agua, donando capital que les garantiza el acceso a la información estratégica y, al tener el conocimiento, cuentan con la posibilidad de orientar las inversiones hacia las fuentes de recursos que, para muchos Estados, hoy ya son escasos.

Los principales interesados en clasificar a los recursos naturales, de manera errada, como capital natural, en vez de patrimonio natural, son las empresas privadas, en especial las multinacionales, que consideran la naturaleza como factor productivo y su preservación como una forma de inversión ligada a los intereses particulares. Bajo esta idea se imponen esos criterios sobre la soberanía de los Estados, mientras sus habitantes sufren el desperdicio y usurpación de sus recursos, especialmente el agua (Arévalo, 2008).

\section{BREVE REVISIÓN DE LA SITUACIÓN DEL AGUA}

Para determinar cuáles son las razones por las que el agua pasó de ser un recurso renovable a uno estratégico y no renovable, es necesario esclarecer qué ha ocurrido con este recurso en razón de su cambio de situación y prioridad en el siglo actual. Foronda (2008) enumeró en cuatro puntos las razones por las cuales se ha dado este cambio:

En primer lugar, por la disponibilidad del mismo, pues solo el 2,5\% del agua en el mundo es potable (Arévalo, 2008); en segundo lugar, por el incremento de la población mundial, la cual ha aumentado en cuarenta la necesidad de agua para diversos fines económicos; en tercer lugar, la mala administración de la misma por cuenta de las malas prácticas humanas como el desperdicio, uso agrícola, fugas, robo y también por la mala gestión; y en cuarto lugar, por la mercantilización del recurso, lo que incrementa los índices de pobreza extrema por la imposibilidad de acceder al servicio.

El agua se ha convertido en un bien excluyente, aún cuando según el derecho internacional, todos los seres humanos tienen derecho a ella. Según el Foro Mundial del Agua (2001), la Conferencia sobre el Agua en Mar de la Plata, Argentina (1977), el Tribunal Latinoamericano del Agua (1998), entre otros, se ha pactado que toda la población debe tener acceso al agua sin importar sus condiciones. El Programa de Naciones Unidas para el Medio Ambiente (PNUMA, 2007), que es la principal autoridad a nivel mundial en el área ambiental, afirma:

"Entre los elementos de la crisis del agua en el mundo se cuentan la duplicación de la población pobre que no tiene suficiente acceso al agua y el saneamiento; la diferencia cada vez mayor entre los ricos y los pobres y las poblaciones urbanas y rurales en los servicios de agua y 
saneamiento; el costo cada vez mayor de los desastres relacionados con el agua; la menor calidad de los recursos hídricos y los ecosistemas; la financiación insuficiente del sector del agua; la mayor presión ejercida sobre los recursos hídricos; la mayor demanda de agua para usos agrícolas e industriales y una mayor contaminación generada por esos usos; y la necesidad de fortalecer la gobernanza del agua".

A partir de la década del setenta, el debate sobre el agua ha tomado dos vertientes fuertemente marcadas y robustecidas por la relevancia que tiene hoy en día el tema ambiental. Una de las vertientes culpa al sistema económico, asumiéndolo como insostenible y causante de la insuficiencia del agua; esta vertiente está compuesta por la sociedad en general, que se muestra inconforme del tratamiento del agua como un bien más que se debe tranzar en el mercado.

La otra vertiente abarca al sector privado, principal interesado en la mercantilización y, por consiguiente, privatización del agua; está a favor del sistema y considera que la escasez se controla a través del mercado, es decir, a través de los precios, los cuales contendrán los excesos en el consumo. Además, acepta que la falta de oferta y la poca eficiencia en la misma se cubra con inversión privada, la cual se dará bajo las condiciones establecidas por los inversionistas (Foronda, 2008).

Según Sergio Abreu (2010) la transformación en la connotación que ha sufrido el recurso agua le ha brindado la capacidad de ser considerado como un factor determinante en la estrategia de la geopolítica internacional, en la que el autor aclara que el problema está en "el agua como un bien común social ligado al derecho a la vida, enfrentado a la definición de la misma como un recurso con valor económico y estratégico, definido además por poderosos grupos económicos y los intereses geoestratégicos de terceros países." Es por ello que las políticas deberían estar enfocadas a alcanzar la conjunción de esta dicotomía, integrando el bienestar social y la explotación óptima del recurso.

Siguiendo el mismo lineamiento, Bellis (2009) afirma que el agua, debido a su característica de recurso limitado, será causa de conflictos a nivel internacional, debido al problema de su distribución y uso ineficiente, el cual ya ha dejado más de un quinto de la población mundial sin acceso a tal recurso. No ha sido gratis la denominación del recurso agua como un bien estratégico si nos atenemos a la idea de posibles conflictos en el corto y mediano plazos. Algunos gobiernos de América latina, con la esperanza de afrontar la crisis y disminuir la escasez, han llevado a cabo prácticas de privatización del recurso que son contradictorias y perjudiciales para la soberanía de las naciones.

La concepción errónea de la naturaleza como capital natural, promovida por las empresas privadas, ha ocasionado que se trate a este recurso como un simple factor de producción, es decir, un motor de ganancia, y a pesar de ser un capital, este no tiene una remuneración, lo que genera una disyuntiva en el concepto, pues es prácticamente imposible calcular el valor de un recurso 
natural. La única forma de valorarlo es como un costo de oportunidad donde, a medida que se agote el recurso, su valor incrementará, generando aún más problemas al momento de calcular su valor. Es por ello que Gudynas (2000) justifica que debe ser tratado como patrimonio natural, entendiendo a este como un recurso que va a prevalecer a través de generaciones, y por este motivo se buscará su conservación.

\section{CONSIDERACIONES TEÓRICAS SOBRE EL TEMA DE LOS RECURSOS NATURALES}

Para direccionar el tema de los recursos naturales es necesario enfocarlo desde la teoría económica, en especial si es un recurso en el cual los derechos de propiedad no están bien definidos, lo cual implica externalidades generadas por el consumo. El resultado se genera por ineficiencias. Una de estas ineficiencias es "la tragedia de los bienes comunales".

El tema de los bienes comunales es un efecto de la mala regulación del uso de recursos comunes, esto genera que el recurso se use excesivamente hasta que los rendimientos de este sean nulos. Cada agente económico busca maximizar su beneficio, pero no tiene en cuenta que todos los demás también van detrás del mismo cometido. Por lo tanto, es necesario evitar que el recurso sea utilizado hasta que los beneficios sean nulos, es decir, consumir hasta que la propensión a contaminar esté por encima a la propensión de renovación y, por consiguiente, se llegue a agotar el recurso (Hardin, 1968).

Siguiendo el mismo lineamiento, Hardin (1968) plantea que la mejor solución a la tragedia de los bienes comunales es la privatización. Sin embargo, también enfatiza en el caso de los recursos naturales, los cuales no pueden ser privatizados, simplemente porque "no podemos cercar el agua y el aire que nos rodea" y, por lo tanto, la tragedia debe ser enfrentada utilizando medios diferentes a la privatización.

Entre las principales soluciones planteadas se encuentran las leyes o los impuestos que afectarían directamente los costos de los agentes, pues el precio de contaminar debe ser negativo y, por consiguiente, cada agente contaminante se debe enfrentar al coste marginal social de sus decisiones. El coste marginal en el cual incurriría un agente reduciendo su contaminación debería ser igual al beneficio marginal que obtiene el agente que se estaba perjudicando por la externalidad (Pigou, 1877-1959, en Varian, 1998).

Así mismo, las soluciones propuestas por Hardin son viables si existe un sistema jurídico para exigir el cumplimiento de esas normas; este puede ser una solución económica para utilizar eficientemente los recursos comunes. Sin embargo, en los casos en los que la ley es ambigua o inexistente, como también cuando las decisiones de carácter legal están en manos de agentes corruptos, en estas situaciones puede surgir fácilmente la tragedia de los bienes comunales. 
Otra posible solución sería la internalización de la externalidad, que se lograría por medio de una negociación entre agentes que tendrían en cuenta la externalidad antes de tomar sus decisiones (Coase, 1960). Sin embargo, esta solución sería viable cuando los costes de transacción, es decir, los costes de llevar a cabo la negociación, sean bajos. En el caso de los recursos estratégicos es más beneficioso contaminar que llegar a un acuerdo, en razón a que para las empresas implica un esfuerzo mayor y una pérdida de utilidad realizar una negociación con los directamente afectados.

Así mismo, para Coase el costo social de la contaminación se puede reducir a una simple negociación entre los afectados y el contaminador. Lo relevante en cualquier caso es tener claridad acerca de los derechos de propiedad sobre el recurso; después de tener claridad sobre ello se facilita llegar a un acuerdo.

\section{RECURSOS NATURALES Y COMERCIO INTERNACIONAL}

La importancia de los recursos estratégicos a nivel internacional se puede explicar desde la teoría de Heckscher-Ohlin (1919; 1924, en Montoya, 2004) donde las diferencias en las dotaciones de recursos entre los países determinan el comercio internacional. En esta teoría el país exportará el recurso, el cual requerirá del factor más abundante y, por consiguiente, más barato. De esta manera resultará más barato para un país con mayor dotación de recursos naturales hacer uso de los mismos para ingresar al comercio internacional.

Por otro lado, un país con abundancia de recursos tenderá a exportarlos a países con ventaja comparativa en tecnología y mano de obra capacitada, y a su vez importará productos de estas naciones (Davis, 2009).

Si se asume de manera hipotética que los recursos naturales hacen parte de mercados con competencia imperfecta, esto los haría propensos a tener poder de mercado, debido a que los recursos naturales se encuentran concentrados en pocas naciones, lo cual limita la capacidad de crecimiento en otros países.

\section{RECURSOS NATURALES Y SOSTENIBILIDAD}

Hotelling (1991) formuló una teoría para explicar el comportamiento de los precios y la tasa de extracción que maximiza el bienestar para los recursos agotables; esta teoría se conoce como "las compensaciones inter-temporales o costos de oportunidad del agotamiento". Este modelo se reconoce por recoger el carácter agotable de los recursos no renovables, y se puede explicar por medio de un mercado de carteles. Por ejemplo, cuando un cartel que comprende recursos naturales abarca todo el mercado de un recurso, este se comportaría 
como un monopolio, el cual podría controlar los precios a nivel mundial del producto en cada momento del tiempo, podría restringir la producción y fijar los precios por encima del coste marginal.

Aunque es posible desde la teoría económica incluir los recursos naturales dentro del mercado por medio de carteles que controlarían la oferta y demanda del recurso, la situación cambia cuando los recursos son de libre acceso conllevando a un aumento en la explotación del recurso de forma excesiva y, de esta forma, a su agotamiento. Bajo esta característica, la explotación sostenible del recurso solo será posible si después de explotarlo se deja un periodo de renovación. Para que se cumpla esta condición, es necesario que el país que cuenta con el recurso tenga un control total sobre el mismo, lo que implica solidez en los derechos de propiedad de cada país, convirtiéndose en la base de una ventaja comparativa (Chichilnisky, 1994). Citado en el Informe sobre el Comercio Mundial de 2010.

También existen fuerzas en la economía que tienen la capacidad de compensar el carácter finito ${ }^{1}$ de los recursos naturales, como son el desarrollo tecnológico y la sustitución de estos por bienes de capital con características similares (Dasgupta y Heal, 1974).

El control y sustitución de los recursos naturales es indispensable desde el punto de vista de la sostenibilidad en razón a que el ritmo de crecimiento de la población y de la producción se ve limitado por la oferta finita de estos recursos, como también por la escasa capacidad de la naturaleza para actuar como depósito de desechos generados por la humanidad.

\section{RECURSOS NATURALES E INTERVENCIÓN ESTATAL}

Del mismo modo, otros autores han demostrado que un país que no cuenta con derechos de propiedad sólidos sufrirá el agotamiento de sus recursos y, por consiguiente, terminará importándolos. Este problema se genera por el precario control que se tiene sobre los recursos y la demanda elevada de los mismos, incluso teniendo grandes existencias de ellos. Los argumentos anteriores muestran que ante una liberalización del comercio, los países deben tener totalmente definidos sus derechos de propiedad sobre las existencias de los recursos (Emami y Johnston, 2000). Citado en el Informe sobre el Comercio Mundial, 2010.

En la realidad, los recursos naturales no están distribuidos equitativamente a través de todos los países. Las economías que cuentan con exceso de recursos naturales no siempre son propensas al crecimiento económico y, en la mayoría de los casos, sufren el efecto contrario. A esta situa-

1. El carácter finito de un recurso implica que su explotación tiene un límite, el cual es el agotamiento de la totalidad del recurso. 
ción se le denomina "la paradoja de la abundancia", la cual, a su vez, puede conllevar al "mal holandés" ${ }^{2}$.

Por otro lado, el debilitamiento de las instituciones puede traer consecuencias negativas a los países que cuentan con ventajas competitivas en los recursos naturales. Se ha demostrado que las instituciones son determinantes para el desarrollo y el crecimiento de las naciones, como también pueden ser obstáculo al crecimiento si tienen estructuras débiles, es decir, un sistema jurídico ineficiente, disyuntivas en la definición de los derechos de propiedad y funcionarios corruptos.

Muchas veces, bajo las condiciones anteriores, las políticas estarán encaminadas a extraer beneficios individuales por encima del bienestar de la sociedad (Bulte y Damania, 2008), como también, cuando incrementan los recursos naturales, las instituciones podrían debilitarse ante el deseo de enriquecimiento.

\section{RECURSOS NATURALES Y CONFLICTOS}

Tal vez, la consecuencia más grave de poseer un exceso de recursos naturales es la propensión a sufrir conflictos civiles, ya sea por grupos rebeldes que buscan obtener provecho de la extracción de los recursos para recaudar dinero y financiar sus costos de funcionamiento, o para generar medidas no favorables para la población, como por ejemplo, la contaminación del ambiente, la migración de la mano de obra, el fenómeno de desplazamiento de sus tierras por estar en zonas aledañas a recursos estratégicos, entre otros. Todos estos ultrajes generan descontento entre la población civil, generando a su vez guerras civiles. (Organización Mundial del Comercio, 2010). Algunos países, especialmente africanos, han sido blanco de colonizaciones por intereses foráneos en razón a la posesión de estratégicos recursos. Colombia no ha sido la excepción y actualmente se puede observar el interés de transnacionales por la explotación de recursos mineros, en especial, oro, plata, platino, carbón y coltan.

\section{CONFLICTOS POR AGUA EN AMÉRICA LATINA}

Ya se explicaron diversas razones por las cuales el agua es considerada hoy en día un recurso estratégico a nivel mundial; así mismo, es evidente que existen diversos países que cuentan con mayores reservas de este recurso; los más favorecidos, como se puede observar en el gráfico No. 1, se encuentran en Asia y América Latina; esta última cuenta con el $26 \%$ del agua potable a

2. Se denomina el "mal holandés" a un incremento en la entrada de divisas a un país, generada por la exportación de un mismo producto, lo cual perjudica al resto de las industrias exportadoras. 
nivel global, la cual abastece a los latinoamericanos (que comprenden un 6\% de la población). Es decir, que es la región con mayor disponibilidad de agua respecto a su cantidad de habitantes en el mundo.

\section{Gráfico No. 1: POBLACIÓN MUNDIAL Y DISPONIBILIDAD DE AGUA POR REGIONES}

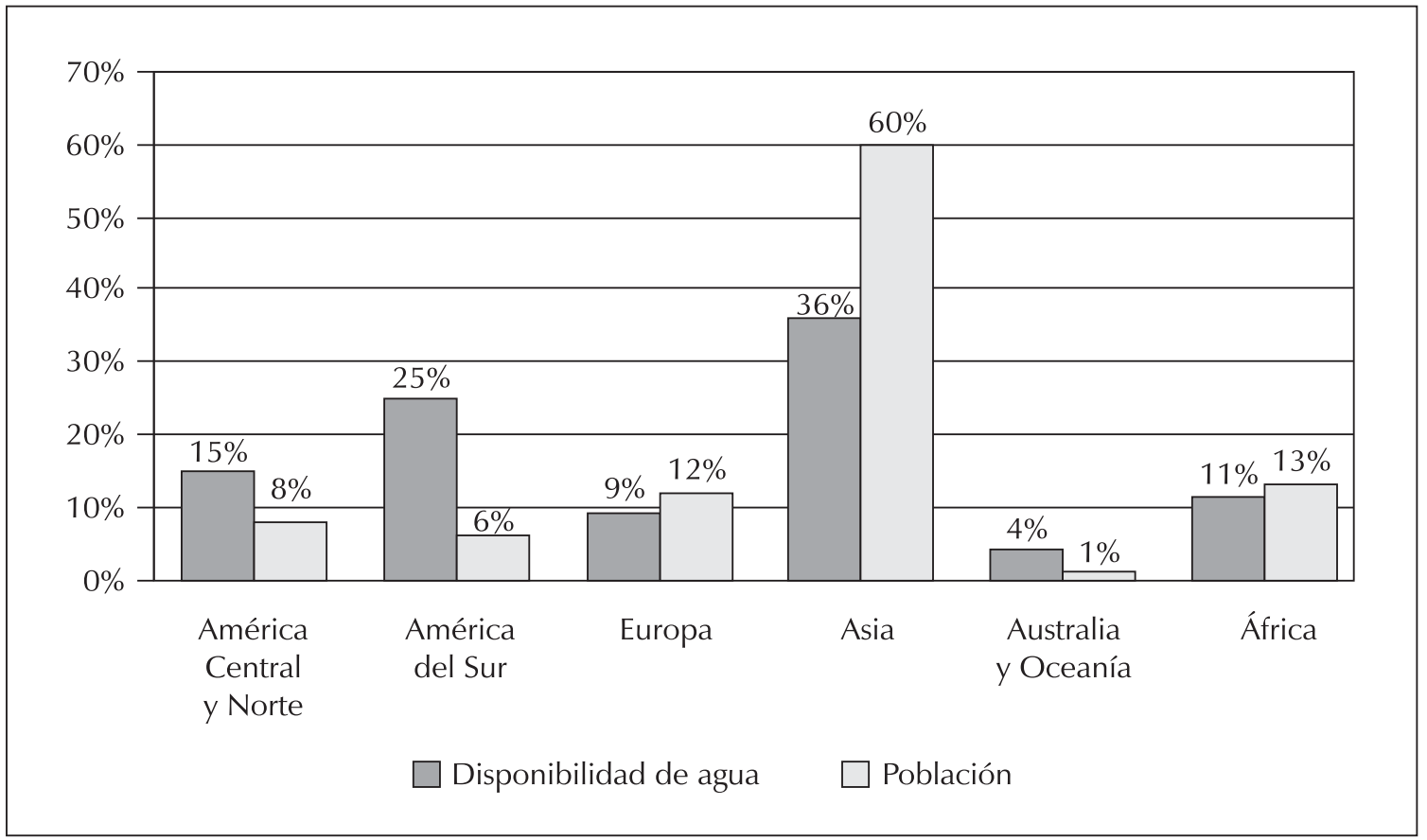

Fuente: International Water and Sanitation Centre (IRC), 2004.

\section{LOS ACTORES DEL CONFLICTO}

América Latina enfrenta un gran inconveniente al poseer tan valioso recurso y, en tal virtud, es acosado permanentemente por diversos actores que hacen parte del conflicto del agua. Estos actores del conflicto se presentan de diferentes maneras y cada cual busca su mayor beneficio. En países en los que los derechos de propiedad no están bien definidos, la ley puede ser ambigua y también puede existir altos niveles de corrupción e ilegalidad que, a la postre, se convierten en un problema de características insospechadas.

Foronda (2008) establece algunos de los actores más relevantes del panorama mundial del agua: en primer lugar está la humanidad, los pueblos que buscan sobrevivir y alcanzar un mayor 
bienestar; en la otra cara están: los gobiernos de cada país, que buscan asegurar su crecimiento; las grandes empresas privadas, que buscan maximizar sus beneficios controlando los recursos naturales y el uso del agua como una mercancía, a fin de sacar provecho de la escasez de la misma; las organizaciones y entidades financieras que ejercen presión sobre los Estados para emplazar sus decisiones y las organizaciones no gubernamentales que defienden el agua.

De los actores anteriores se puede hacer una subdivisión de la siguiente manera: entre los Estados se pueden encontrar los que poseen un recurso y los que no lo poseen, estos últimos quieren tener poder sobre el mismo, por fines estratégicos. Entre las empresas privadas, aunque todas tienen como finalidad lucrarse del agua, su explotación se da en diferente proporción y cada una afecta en distinta medida a la población; entre las más relevantes encontramos las embotelladoras, privatizadoras de empresas públicas y los represadores y desviadores del agua para producción de energía eléctrica. Entre las organizaciones internacionales se encuentran las que están a favor de la comercialización del agua y las que la ven como un bien común.

Los conflictos por agua se pueden dar desde cualquiera de las subdivisiones anteriores o pueden ser una combinación de las mismas. Los conflictos se dan especialmente en naciones que no cuentan con una independencia en sus políticas, donde su legislación es ambigua o inexistente, porque sufren de dependencia económica de otros países y cuentan con puntos débiles a nivel social, cultural o económico que les hace blanco fácil para manipulaciones por parte de organizaciones y bancos internacionales. Este es el caso de América Latina.

\section{EL PAPEL DE ALGUNOS ORGANISMOS INTERNACIONALES RESPECTO A LAS ZONAS CON RECURSOS HÍDRICOS ESTRATÉGICOS}

Las aguas subterráneas o acuíferos comprenden el 98\% de agua dulce que existe en el planeta. América Latina cuenta con el tercer acuífero con mayor volumen de agua a nivel mundial. Este acuífero podría abastecer de agua a 6.000 millones de personas durante 200 años.

Esta fuente, que recibe el nombre de Acuífero Guaraní, está ubicada entre cuatro países latinoamericanos: Brasil, Argentina, Paraguay y Uruguay. Y las decisiones tomadas respecto a la regulación de este recurso hacen parte de las consideraciones de los gobiernos del MERCOSUR, los cuales han hecho especial énfasis en la defensa de la soberanía de los territorios y reservas de agua tanto superficiales como subterráneas (Iglesias \& Taks, 2007).

Contrario a lo que opinan los gobiernos de los países que cuentan con parte de la gran reserva de agua subterránea, las organizaciones internacionales hacen énfasis en que los acuíferos transfronterizos son "una oportunidad de cooperación internacional". Cooperación que se ha ido incrementando por el gran interés que existe a nivel internacional sobre el recurso (Miletto, 2004). 
La "cooperación" se ha dado por medio de financiación de un proyecto para determinar qué tan utilizable es el agua que contiene el acuífero. Los 27 millones de dólares necesarios para Ilevar a cabo el proyecto provienen en gran parte del Global Enviroment Facility (13,4 millones), cuyos recursos son controlados por el Banco Mundial y el resto del dinero es financiado por diversos organismos como la Organización Internacional de Energía Atómica, el Servicio Geológico de Alemania, el Programa de Recursos Hídricos del Banco Mundial, la Organización de los Estados Americanos y el Gobierno de los Países Bajos (Delgado, 2004).

También se puede encontrar intervención del Banco Mundial, el Banco Interamericano de Desarrollo, entre otros organismos internacionales, y empresas privadas en otras fuentes estratégicas de recursos hídricos como la cuenca de la Plata (acuífero que surte de agua a un cuarto del continente sudamericano y, a su vez, hace parte de la zona del MERCOSUR), entre otros acuíferos del continente latinoamericano.

La especial preocupación de estos organismos sobre la conservación y uso sustentable de los recursos no es más que otra iniciativa de su modelo neoliberal, que por lo general significa la privatización de tales recursos con el fin último de generar grandes ganancias a algunas empresas privadas, las cuales "cumplen con los requisitos" establecidos por el BM, pero en realidad no tienen mayor interés en asegurar la conservación del medio ambiente. Lo anterior conllevaría al inconformismo de la población procurando la conformación de movimientos de resistencia civil que podrían terminar en guerras civiles.

\section{MILITARIZACIÓN DE LAS ZONAS CON RECURSOS HÍDRICOS ESTRATÉGICOS}

En las zonas donde se ubican los acuíferos de América Latina no solo se encuentran los organismos internacionales y las empresas privadas multinacionales privatizadoras de los servicios de agua, también podemos encontrar bases militares del Ejército de los Estados Unidos de América.

Es evidente para la mayoría de los países que para el año 2025, cuando la demanda de agua esté un 56\% por encima de la oferta, esta será determinante para el control del poder a nivel mundial. Lo anterior no es un secreto para los Estados Unidos, por este motivo los países que cuentan con este recurso se convertirán en víctimas del intervencionismo. Una de las principales excusas del mismo es el terrorismo (Revilla, 2005).

En el caso de los acuíferos latinoamericanos se puede evidenciar cómo se justifica el intervencionismo por medio de un reporte presentado por la Federal Research Division (2003, en Ebid.), en el cual presentan algunas de las siguientes conclusiones:

Los gobiernos de los países miembros de la triple frontera (donde se ubica el acuífero) están facilitando actividades de grupos terroristas islámicos; la capacidad militar de los países de la 
triple frontera es inadecuada para afrontar los grupos terroristas islámicos; la legislación de los gobiernos y la corrupción favorecen la recaudación de fondos para el terrorismo y actividades terroristas. Sin embargo, aunque aún no se ha comprobado la existencia de tales "grupos terroristas islámicos", ya se han instalado bases militares de E.U. en la triple frontera y en diversas fuentes de recursos hídricos alrededor de América Latina.

Las bases militares no solo están ubicadas de forma estratégica a través de todo el continente, sino que también están sobre las grandes fuentes de recursos hídricos. Un reciente Tratado, considerado inviable por el Congreso de Colombia, aceptó la intervención de técnicos y asesores militares norteamericanos en siete bases militares colombianas. A su vez, cerca de las bases militares se pueden encontrar diversos movimientos sociales de resistencia. Tan solo en la zona del Acuífero Guaraní, que a su vez está cercano a la cuenca de la Plata, se ubican dos bases militares y tres movimientos sociales de resistencia.

Los movimientos sociales básicamente son grupos conformados por la sociedad civil que se ve afectada debido a los procesos de neocolonialismo que se llevan a cabo en las zonas donde habitan. Estos movimientos van en contra de los intereses de las transnacionales que puedan afectar su bienestar. Así mismo, estas últimas encargadas ya sea de privatizar los servicios, construir represas, hidroeléctricas, entre otros, buscan básicamente maximizar sus ganancias, en la mayoría de los casos, favorecidas por la corrupción en las instituciones públicas.

Aunque los miembros de los movimientos de resistencia social solo buscan asegurar las condiciones básicas para su bienestar y subsistencia, los Estados se encargan de reprimir los movimientos de resistencia; esta represión, en la mayoría de los casos, se da por medios violentos. Sin embargo, estos movimientos sociales se dan precisamente por la indiferencia del Estado y surgen como medio para llamar la atención del mismo ante las necesidades sociales.

Cuando el Estado se empecina en proteger los intereses extranjeros se puede agravar el conflicto hasta llegar a la matanza de líderes campesinos y la criminalización de la protesta (Acuña, 2005). A su vez, estos conflictos pueden convertirse en razones para fortalecer el intervencionismo de otras naciones, las cuales justifican la militarización de la zona por la existencia de movimientos terroristas que, en algunos casos, pueden suplantar a los movimientos de resistencia social.

\section{CONFLICTOS DERIVADOS DE LA PRIVATIZACIÓN}

La distribución del agua es una cuestión democrática, pues incide en el bienestar de cada uno de los miembros de la población; por consiguiente, debería ser el Estado el encargado de captar las necesidades de la población y llevar a cabo una gestión de los recursos hídricos de tal forma que estos lleguen de manera eficaz y con ciertos estándares de calidad. Es la población la que quiere decidir sobre su futuro y su bienestar; por consiguiente, en la mayoría de los casos, la privatización genera desacuerdo a nivel social. 
Sin embargo, para asegurar esta gestión se requieren recursos, los cuales solo se pueden obtener mediante endeudamiento. Pero los organismos internacionales que facilitan tales recursos no están a favor de la gestión estatal y sus requisitos favorecen a las empresas privadas. El modelo neoliberal de tales organismos internacionales se basa en la premisa de la ineficiencia del Estado y la incapacidad del mismo para satisfacer las necesidades de la población. Sin embargo, se ha comprobado por la experiencia que muchas veces es mejor la gestión estatal (Farro, 2008).

A continuación se presentarán algunos casos en los cuales la privatización ha causado problemáticas a nivel social que han generado disturbios y enfrentamientos sociales como también contiendas en el ámbito internacional.

\subsection{Guerra civil por agua: el caso de Bolivia}

El primer gran conflicto por agua en América Latina se dio en la ciudad de Cochabamba, Bolivia, durante el año 1999 por la presión del BM, en virtud de un ajuste estructural, o privatización del servicio de agua. El servicio de agua fue transferido a Aguas del Tunari, que a su vez era una subdivisión de International Water (Hall \& Lobina, 2002).

Para ese mismo año las tarifas del agua se incrementaron hasta en $200 \%$, lo cual genero un caos a nivel social en el año 2000. El conflicto se incrementó por la represión militar ejercida por el Estado, lo cual dejó un saldo de un muerto y varios heridos. Ante la situación social, Aguas del Tunari revocó la concesión y para el año 2001 presentó una demanda de indemnización por 25 millones de dólares ante el Centro Internacional de Arreglo de Diferencias Relativas a Inversiones (CIADI) del Banco Mundial.

En este mismo país también se presentó otro caso de privatización que a su vez derivó en protestas por parte de la sociedad civil, debido al incumplimiento de las metas de ampliación del servicio en las zonas de El Alto y La Paz. La privatización la asumió la transnacional Aguas de Illimani, la cual detuvo las obras de ampliación de cobertura, pues no estaba obteniendo los rendimientos esperados. Ante tal situación se formaron diversos movimientos sociales, los cuales, respaldados por movilizaciones, decretaron paro durante tres días consecutivos protestando por las dificultades con el servicio de agua prestado por la transnacional (Farro, 2008).

\subsection{Tráfico y contaminación del agua: el caso de Brasil}

Sanepar, la compañía de agua del Estado de Paraná, Brasil, fue privatizada en 1998. Después de la privatización se empezó a incrementar el número de habitantes con padecimientos de tipo renal. Al realizar estudios de laboratorio se comprobó que el agua que llegaba a las viviendas estaba contaminada. Debido a los daños que causó Sanepar a la población por poner en peligro 
la salud pública, una ONG, llamada Instituto Tomoneira, tuvo la tentativa de demandar a la empresa exigiendo una indemnización y el encarcelamiento del director de operaciones. En otras zonas donde esta empresa suministraba el servicio de agua también se presentaban casos en los que no cumplía con las normas básicas de saneamiento (Hall \& Lobina, 2002).

Además del mal servicio prestado por la transnacional, esta se encontraba realizando actividades ilícitas dentro del país, como el tráfico de agua, la cual era extraída directamente de acuíferos subterráneos, sin ningún permiso ni gubernamental ni ambiental. La extracción ilegal de agua ha generado derrumbes en la zona, los cuales han afectado directamente a la población, como también se ha comprobado que la empresa vierte aguas contaminadas en los ríos (Hall \& Lobina, 2002).

Aunque existen más casos en los cuales se corroboran las ineficiencias derivadas de la privatización de las empresas públicas que prestan el servicio de agua en América Latina, no es el tema central de este trabajo presentar todos los casos de privatizaciones del servicio de agua en esta región ${ }^{3}$. Sin embargo, los casos específicos de Bolivia y Brasil hacen más explícitas las problemáticas que se pueden generar debido a la privatización.

\section{CONCLUSIONES}

Teniendo en cuenta lo presentado en los apartados anteriores se puede evidenciar un interés por parte de América Latina de preservar y mantener la soberanía ${ }^{4}$ sobre sus recursos estratégicos. Lo cual se ha buscado alcanzar en el marco de las integraciones intra-regionales, como es el caso del Mercosur. Los países miembros de esta integración han demostrado interés en la preservación y regulación de los recursos hídricos. Sin embargo, sus esfuerzos de regulación se han dado recientemente y, por consiguiente, no hay claridad frente a las políticas y legislación que se quiere ejercer sobre los recursos.

Estos países no cuentan con el capital suficiente para evaluar la capacidad de las grandes reservas de estos recursos, lo cual conlleva nuevamente a la intervención de los organismos internacionales que, a su vez, traen consigo a las transnacionales, cuyo objetivo consiste en maximizar

3. Para mayor información acerca de casos de privatización del servicio de agua en América Latina, ver (Hall \& Lobina, 2002).

4. Entre otras situaciones que pueden ser posibles detonantes de conflictos por recursos estratégicos en América Latina se encuentran los proyectos para crear "corredores de infraestructura", como por ejemplo, el lirsa, el PPP y el Nawapa. Estos proyectos buscan la movilización de grandes cantidades de recursos naturales a través de toda América, pero especialmente desde América del Sur y Central hacia América del Norte. Vale la pena investigar respecto a este tema, debido a que implica la extracción directa de los recursos de la región y la inversión de grandes cantidades de capital, a fin de movilizar tales recursos. Para mayor información ver (Delgado, 2004). 
sus ganancias, generando problemáticas sociales que culminarán en conflictos o movimientos sociales de resistencia. Estos últimos servirán en ciertos casos como justificación para que las naciones interesadas en los recursos usen el intervencionismo justificándose en la prevención de la conformación de grupos terroristas, todo a fin de establecer bases militares sobre los recursos estratégicos.

Así es la forma como se conglomeran tantos entes extranjeros alrededor de los recursos hídricos estratégicos, violando los objetivos de soberanía y de regulación que en un principio tenían los países latinoamericanos.

La problemática que enfrentan los países de América Latina referente a los recursos estratégicos, especialmente el agua, implica diversos aspectos y cada uno de ellos es de algún modo un detonante de conflicto. Los principales afectados por estos conflictos, indudablemente, son los habitantes de la región, golpeando con más fuerza a la población pobre.

Los conflictos por agua, mencionados en este trabajo, giran en torno a tres temáticas: en primer lugar, la privatización del servicio de agua potable y alcantarillado, medida que puede ser perjudicial para la población si el Estado no evalúa las posibles consecuencias de la misma, como también puede acentuar la brecha de pobreza y generar problemas de salubridad que a su vez generarán inconformismo a nivel social provocando la creación de movimientos sociales de resistencia.

En segundo lugar, el intervencionismo de potencias extranjeras interesadas en establecerse cerca a las zonas estratégicas donde se encuentran las grandes fuentes y reservas de agua, valiéndose de afirmaciones infundamentadas, como la existencia de grupos terroristas en las zonas cercanas a las grandes reservas de recursos estratégicos.

En tercer lugar, los intereses de los organismos internacionales, que promueven la privatización, convirtiendo a las transnacionales en las principales beneficiadas de la distribución del agua potable, mientras la sociedad civil sufre las consecuencias de la privatización y los estados quedan exentos de su soberanía, viéndose obligados a acudir a los estrados internacionales a fin de hacer valer los derechos de la población.

Se hace necesario profundizar en cada uno de los temas presentados en el presente documento, pues es indispensable esclarecer cuál es la situación actual de América Latina respecto a la administración de sus recursos y los obstáculos que debe afrontar en el proceso de ejercer un explícito derecho de propiedad y una clara legislación sobre los mismos, basados en las situaciones ya afrontadas y previendo las futuras problemáticas. 


\section{BIBLIOGRAFÍA}

- Varian, Hal. (1998). Microeconomía intermedia. Barcelona: Antoni Bosch Editor.

- $\quad$ Abreu, S. (2010, junio). El agua: un recurso estratégico. Consejo Uruguayo para las Relaciones Internacionales, informe Técnico, № 3, Uruguay.

- Bellis, B. Q. (2009). El agua: recurso vital de las civilizaciones. En Obras Sanitarias del Estado (Eds.). Conferencia. Evento internacional en el "Día Mundial del Agua. Uruguay.

- Gudynas, E. (2000). Los límites de la sustentabilidad débil, y el tránsito desde el capital natural al patrocinio ecológico. En Centro Latino-Americano de Ecología Social (Eds.) Educación, Participación y Ambiente. (p. 4: 7-11).

- Sandoval, L. R. (2001). El concepto de capital natural en los modelos de crecimiento exógeno. Análisis Económico, XVI (033), 109-128.

- Serrano, J. A. (2007). Geopolítica del agua en América Latina: dependencia, exclusión y privatización. Documento de trabajo. En XVI Simposio Polaco-Mexicano. Varsovia.

- Montoya, G. (2004). Estado actual de la teoría Heckscher-Ohlin. Tesis de maestría no publicada. Bogotá, Universidad Nacional de Colombia, Facultad de Ciencias Económicas.

- Davis, G. (2009). Trade in mineral resources. Ginebra, Suiza: Colorado School of Mines, Economic Research and Statistics Division.

- Hotelling, H. (1991). The economics of exhaustible resources. Bulleting of Mathematical Biology, 53(1/2), 281-312.

- Dasgupta, P.S. \& Heal, G. (1974). The optimal depletion of exhaustible resources. Review of Economic Studies, 4, 3-28.

- Organización Mundial del Comercio. (2010). Informe sobre el comercio mundial: el comercio de recursos naturales. Ginebra, Suiza.

- Bulte, E. H. \& Damania, R. (2008). Resources for sale: Corruption, democracy and the natural resource curse. The B.E. Journal of Economic Analysis \& Policy, 8.

- Acuña, I. T. (2005). TLC, Movimientos sociales y futuro de la Amazonia colombiana. Revista Luna Azul, Universidad de Caldas, 25.

- Delgado, G. C. (2004). IIRSA y la ecología política del agua sudamericana. Tesis de grado no publicada. Barcelona, Universidad Autónoma de Barcelona, Instituto de Ciencias y Tecnología Ambiental.

- Farro, M. E. (2008). El agua como derecho humano. El caso de América Latina. Estudios para la paz, Fundación Seminario de Investigación para la Paz, 22, 297-343. 
- Hardin, G. (1968). The tragedy of the commons. Science, 162 (3859),1243-1248.

- Iglesias, V., \& Taks, J. (2007). Acuífero Guaraní, por una gestión participativa. Voces y propuestas desde el movimiento del agua. Montevideo: Casa Bertolt Brecht.

- Organización de los Estados Americanos. (2004). El recurso invisible. Acuíferos transfronterizos: una oportunidad de cooperación internacional. 3.

- Programa de las Naciones Unidas para el Medio Ambiente (Pnuma). (n.d.). Estrategia y política del agua del PNUMA. Panamá: PNU. Extraido Marzo 30, 2011 desde http://www.unep.org/Themes/ freshwater/ Documents/Water_Policy_Strategy_spanish.pdf.

- Revilla, P. C. (2005). Consecuencias jurídico-políticas de la invasión a Iraq: reformulación del sistema mundial actual. Una visión evolutiva. En: Sánchez, C. (Eds.). Aspectos jurídico-políticos de la guerra en Iraq. México: Universidad Nacional Autónoma de México.

- Sánchez, L. D., \& Sánchez Torres, A. (2004). Uso eficiente del agua. Países Bajos: International Water and Sanitation Centre. 